\title{
3EG J2027+3429: Another blazar behind the Galactic Plane
}

\author{
V. Sguera ${ }^{1}$, A. Malizia², L. Bassani² , J. B. Stephen ${ }^{2}$, and G. Di Cocco ${ }^{2}$ \\ 1 Dipartimento di Astronomia di Bologna, via Ranzani 1, 40127 Bologna, Italy \\ 2 Istituto di Astrofisica Spaziale e Fisica Cosmica Sezione di Bologna, Area di Ricerca di Bologna, Via P. Gobetti 101, \\ Bologna, Italy
}

Received 21 May 2003 / Accepted 19 August 2003

\begin{abstract}
We report on the association of an X-ray source (WGA J2025.1+3342), serendipitously found with BeppoSAX in two separate observations, with the unidentified EGRET source 3EG J2027+3429. The source is detected from $1 \mathrm{keV}$ up to about $100 \mathrm{keV}$, has a flat $(\Gamma=0.6-1.5)$ spectrum and is highly variable both in intensity and shape. The data indicate marginal evidence for an iron line in the source rest frame. The overall X-ray luminosity is $\sim 4 \times 10^{45} \mathrm{erg} \mathrm{s}^{-1}$, typical of a quasar. The $\mathrm{X}$-ray source is coincident in radio wavelengths with a bright object characterized by a flat spectrum over the band $0.3-10 \mathrm{GHz}$ while in the optical it is identified with a quasar at redshift 0.22. All available data indicate a SED compatible with a low frequency peaked or red blazar type object. This identification is interesting because this is the second blazar found behind the galactic plane in the direction of the Cygnus region.
\end{abstract}

Key words. X-rays: galaxies - X-rays: individual: 3EG J2027+3429 - BL Lacertae objects: general

\section{Introduction}

The nature of unidentified gamma-ray sources has remained a mystery since the first survey of the gamma-ray sky with the COS-B satellite. More than sixty percent of the 271 high energy sources reported in the 3rd EGRET Catalogue (Hartman et al. 1999) are still unidentified, with no firmly established counterparts at other wavebands. Considering their distribution on the sky, we can infer that one third of these are extragalactic (probably AGNs of the blazar type), the rest most likely being objects within the Milky Way. Most of the AGN blazars are strong flat spectrum radio sources (i.e. jet dominated) and are variable in the gamma-ray band. Of the galactic population, steady sources are likely to be radio-quiet pulsars, while transient objects are still poorly understood and may be due to interactions of individual pulsars or neutron star binaries with the ambient interstellar medium. The main difficulty in identifying EGRET sources is their often large error box; therefore a positional correlation with a known object is usually not enough to identify a source. For this reason, a multiwavelength approach, using X-ray, optical and radio data, is often needed to understand the nature of these sources (see Caraveo 2002 and references therein). Searches for X-ray counterparts, especially at high energies, are particularly useful in finding a positionallycorrelated, highly unusual object with special parameters that might be expected to produce gamma rays. For this reason we have recently started a program to search the BeppoSAX archive for observations covering either partially or totally EGRET error boxes (Sguera et al. 2003). During this search, we

Send offprint requests to: V. Sguera, e-mail: sguera@bo.iasf.cnr.it discovered a hard X-ray source within the $99 \%$ confidence error box of 3EG J2027+3429 (also known as 2EG J2026+3610). The gamma-ray source is at a low galactic latitude in the Cygnus region and has recently been proposed as being associated with an extragalactic object on the basis of a radio search and optical follow up observations (Sowards-Emmerd et al. 2002). Previous works have suggested a young pulsar as a possible counterpart, given the presence in the EGRET error box of an OB star association (Zhang \& Cheng 2000; Romero et al. 1999) although the gamma-ray flux variability and spectral characteristics (Merck et al. 1996) argue against this possibility. The extragalactic interpretation is instead more attractive given the nature of the proposed optical counterpart (a Flat Spectrum Radio Quasar or FSRQ) and variability of the gamma-ray emission, although this would be unusual given the proximity of the source to the Galactic Plane. We show in the present paper that the extragalactic optical/radio source is also an X-ray emitter from $0.2 \mathrm{keV}$ to $\sim 100 \mathrm{keV}$ and furthermore that it displays variability at these frequencies. Our X-ray data together with an overall assessment of the source's spectral characteristics confirm the blazar nature of this object and further indicate that now 2 such sources have been found at low galactic latitudes after the identification of 3EG J2016+3657 as a blazar-like radio source G74.87+1.22 (Mukherjee et al. 2000; Halpern et al. 2001).

\section{Search for X-ray counterparts in the EGRET error box}

The X-ray counterpart of 3EG J2027+3429 was found in two BeppoSAX-MECS observations targeted towards the black 




Fig. 1. The BeppoSAX/MECS (2-10 keV) image superimposed on the EGRET $\gamma$-ray probability contours at $50 \%, 68 \%, 95 \%, 99 \%$ confidence level. All the X-ray counterparts listed in Table 1 are also shown: pluses are WGA sources, crosses are ROSAT faint sources and squares are the ROSAT bright sources.

hole candidate GS2023+338 (also V404 Cygni). In Fig. 1, one of these two BeppoSAX-MECS (2-10 keV) images is superimposed on the EGRET $\gamma$-ray probability contours $(50 \%, 68 \%$, 95\% and 99\%). The black hole candidate lies just outside these contours which makes it less likely to be the X-ray counterpart of the EGRET source. Instead, another source, also well visible in the $2-10 \mathrm{keV}$ image, is located within the $99 \%$ con-

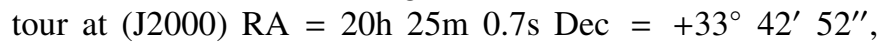
25 arcmin north-east of the target source (the uncertainty associated with the source position is 1 arcmin, $90 \%$ confidence level). The source is visible in both MECS observations but is only marginally detected in the only available LECS image, thus indicating a hard X-ray spectrum.

GS2023+338 was also observed by the ASCA satellite but unfortunately the EGRET candidate counterpart is at the edge of the GIS telescope field of view and although it is well detected, the data are too affected by vignetting to provide useful information on its spectral shape (HEASARC database).

There is also a pointed ROSAT observation of the black hole candidate, which provides a total of $17 \mathrm{X}$-ray sources reported in the WGA catalogue (White et al. 1994). Furthermore, the cross correlation of the 99\% EGRET error box with the ROSAT All-Sky-Survey catalogues (Bright and Faint) resulted in 5 objects, 3 of which are also WGA sources. Therefore all together there are $19 \mathrm{X}$-ray ROSAT sources within the EGRET error box. All these soft X-ray candidates are listed in Table 1 together with their coordinates, ROSAT count rates and offset from the 3EG source position. Most of these objects are unidentified in the Simbad/NED databases except for 6 objects which are associated with normal stars (sources No. 4, No. 9, No. 15, No. 19, No. 20). Only one source, No. 13 (WGA J2025.1+3342) in Table 1, is detected at energies greater than a few $\mathrm{keV}$ and it is the source coincident with the object serendipitously detected by BeppoSAX and ASCA. The ROSAT count rate of WGA No. 13 provides a crude flux estimate of $\sim 10^{-13} \mathrm{erg} \mathrm{cm}^{-2} \mathrm{~s}^{-1}$ in the $0.05-2 \mathrm{keV}$ band, compatible with the marginal detection by BeppoSAX at low energies given the low exposure of the LECS instrument.

A cross correlation of all X-ray objects with radio catalogues available from the HEASARC database indicates that only 2 objects are radio emitting: WGA No. 11 (also RXS No. 22) and WGA No. 13. These radio sources have a $20 \mathrm{~cm}$ flux of 5 and $1268 \mathrm{mJy}$ respectively and therefore the first object is not sufficiently bright to be a likely counterpart of a gamma-ray source (see Sect. 4). All other radio sources detected within the EGRET 99\% error contour are also all too faint (all their fluxes are below $200 \mathrm{mJy}$ at 1.5 and $5 \mathrm{GHz}$ ) to be likely associations of the gamma-ray source.

To conclude, WGA No. 13 is not only the strongest hard $\mathrm{X}$-ray source in the EGRET error box but it is also the brightest radio object. In optical, this source was recently observed by Sowards-Emmerd et al. (2002) who obtained the first spectrum and measured a redshift of $z=0.22$. The spectrum shows emission lines of the Balmer series and so the source can be optically classified as a Quasar.

\section{BeppoSAX observations of WGA J2025.1+3342}

BeppoSAX-NFI pointed at GS 2023+338 in September 1996 and December 2000, for an effective MECS exposure time of 21 and 48 ks respectively; no LECS data are available for the first observation while the second has an exposure of only $6 \mathrm{ks}$. The PDS exposures are instead 10 and $21 \mathrm{ks}$ for the first and second pointings. Standard data reduction was performed on the BeppoSAX data using the software package "SAXDAS" (Fiore et al. 1998). The reduction procedures and screening criteria used to produce the linearized and equalized (between the two MECS) event files were standard and took into account the offset position of the source. For the PDS data we adopted a fine energy and temperature dependent Rise Time selection, which decreases the PDS background by 40\%, improving the signal to noise ratio of faint sources by about 1.5 (Frontera et al. 1997). Spectral fits were performed using the XSPEC 11.0.1 software package and public response matrices for the off-axis sources as from the 1998 November issue. PI (Pulse Invariant) channels were rebinned in order to sample the instrument resolution with the same number of channels at all energies when possible and to have at least 20 counts per bin. This allows the use of the $\chi^{2}$ method in determining the best fit parameters, since the distribution in each channel can be considered Gaussian. In the following analysis, we use an absorbed component to take into account the high galactic column density that in this direction is $7.7 \times 10^{21} \mathrm{~cm}^{-2}$ (obtained from $21 \mathrm{~cm}$ radio data provided by XSPEC). The quoted errors correspond to $90 \%$ confidence level for one interesting parameter $\left(\Delta \chi^{2}=2.71\right)$. The $\mathrm{X}$-ray candidate for the 3EG J2027+3429 source is well detected in the $2-10 \mathrm{keV}$ energy range at $13 \sigma$ and $9 \sigma$ level but it is barely visible in the only 
Table 1. ROSAT sources in the EGRET error box.

\begin{tabular}{|c|c|c|c|c|c|c|c|}
\hline & Source & $\begin{array}{c}\text { RA } \\
(\mathrm{J} 2000)\end{array}$ & $\begin{array}{c}\text { Dec } \\
\mathrm{J}(2000)\end{array}$ & $\begin{array}{c}\text { Count rate } \\
\mathrm{Cts} / \mathrm{s}\end{array}$ & $\begin{array}{c}\text { Search offset } \\
\text { arcmin }\end{array}$ & Type & Radio count. \\
\hline 1 & 1WGA J2024.4+3437 & 202425.90 & +343729.0 & 0.0135 & 31.542 & - & No \\
\hline 2 & 1WGA J2024.5+3422 & 202431.32 & +342244.0 & 0.0060 & 31.854 & - & No \\
\hline 3 & 1WGA J2024.3+3445 & 202418.90 & +344548.0 & 0.0275 & 35.057 & - & No \\
\hline 4 & 1WGA J2025.4+3403 & 202528.40 & +340306.0 & 0.0017 & 35.119 & - & No \\
\hline 5 & 1WGA J2024.1+3418 & 202408.20 & +341850.0 & 0.0024 & 37.692 & - & No \\
\hline 6 & 1WGA J2026.2+3352 & 202617.30 & +335252.0 & 0.0022 & 40.982 & - & No \\
\hline 7 & 1WGA J2024.2+3406 & 202415.90 & +340640.0 & 0.0046 & 42.523 & - & No \\
\hline 8 & 1WGA J2026.0+3350 & 202603.60 & +335024.0 & 0.0032 & 44.041 & - & No \\
\hline 9 & 1WGA J2027.6+3344 & 202738.00 & +334441.0 & 0.0080 & 49.036 & - & No \\
\hline 10 & 1WGA J2026.3+3343 & 202621.20 & +334354.0 & 0.0056 & 49.676 & - & No \\
\hline 11 & 1WGA J2026.9+3343 & 202657.50 & +334308.0 & 0.0918 & 49.873 & - & Yes \\
\hline 12 & 1WGA J2026.3+3340 & 202619.00 & +334050.0 & 0.0066 & 52.775 & - & No \\
\hline 13 & 1WGA J2025.1+3342 & 202510.50 & +334300.0 & 0.0070 & 54.691 & - & Yes \\
\hline 14 & 1WGA J2025.9+3339 & 202559.60 & +33 3914.0 & 0.0089 & 55.088 & - & No \\
\hline 15 & 1WGA J2024.3+3347 & 202423.50 & +334716.0 & 0.0009 & 55.741 & - & No \\
\hline 16 & 1WGA J2024.9+3338 & 202455.00 & +333805.0 & 0.0016 & 60.495 & - & No \\
\hline 17 & 1WGA J2026.5+3330 & 202635.00 & +333039.0 & 0.0098 & 62.526 & - & No \\
\hline 18 & 1RXS J202427.2+343641 ${ }^{(a)}$ & 202427.19 & +343641.0 & 0.0215 & 31.177 & $\mathrm{~F}$ & No \\
\hline 19 & 1RXS J202550.8+350938 & 202550.80 & +350938.5 & 0.0144 & 39.120 & $\mathrm{~F}$ & No \\
\hline 20 & 1RXS J202635.2+331758 & 202635.20 & +331758.5 & 0.0168 & 75.169 & $\mathrm{~F}$ & No \\
\hline 21 & 1RXS J202422.2+344631 $1^{(b)}$ & 202422.20 & +344631.5 & 0.0500 & 34.699 & B & No \\
\hline 22 & 1RXS J202658.5+334253 $3^{(c)}$ & 202658.50 & +334253.0 & 0.0600 & 50.117 & B & Yes \\
\hline
\end{tabular}

${ }^{(a)}$ also a WGA source: 1WGA J2024.4+3437 (source No. 1 in the table). ${ }^{(b)}$ also a WGA source: 1 WGA J2025.4+3445 (No. 3 in the table). ${ }^{(c)}$ also a WGA source: 1WGA J2026.9+3343 (No. No. 11 in the table).

available LECS image. At higher energy, in the PDS regime, a $4 \sigma$ detection was measured only in the second observation.

The PDS instrument has no imaging capability and a field of view of $1.3^{\circ}$ (FWHM), hexagonal in shape. Therefore any object within this field of view can be responsible for the high energy emission. As a first step it is natural to attribute the PDS emission to the black hole candidate; we therefore checked if this is indeed true by performing a spectral analysis of the combined MECS data of GS2023+338 with high energy PDS data. The source was quiescent during the 2000 BeppoSAX observation and its X-ray spectrum was well represented by a power law $(\Gamma \sim 1.9)$ absorbed by the galactic column density (Campana et al. 2001). A fit with this model to the combined MECS/PDS data sets provides a value of $15_{-8}^{+11}$ for the cross calibration constant between the two instruments; this value is well outside the nominal range of $0.75-0.95$ reported by Fiore et al. (1998). Furthermore, extrapolating into the PDS (20-100 keV) energy band, the low energy power law gives a flux of $6.1 \times 10^{-13} \mathrm{erg} \mathrm{cm}^{-2} \mathrm{~s}^{-1}$ which is below the measured PDS flux. Both these facts strongly indicate that the PDS emission either is not due to the black hole candidate or is at least highly contaminated by the presence of another $\mathrm{X}$-ray source in the PDS field of view. We have searched the HEASARC data base for other possible high energy emitting sources located within the PDS field of view but have found none that can be considered a likely hard X-ray emitter.

We therefore assume that the high energy emission, or at least the major part of it, comes from the X-ray counterpart of the $3 E G \mathrm{~J} 2027+3429$ object; this is reasonable given the hard $\mathrm{X}$-ray spectrum of the source compared to the black hole candidate (see below). Consequently we fitted contemporaneously the MECS data from this new source and the PDS points as performed for GS2023+338.

The $2-10 \mathrm{keV}$ spectrum of the first observation is simply fitted with a power law of photon index $\Gamma=1.41_{-0.41}^{+0.45}$ (once galactic absorption is accounted for) and provides a $2-10 \mathrm{keV}$ unabsorbed flux of $\sim 3 \times 10^{-12} \mathrm{erg} \mathrm{cm}^{-2} \mathrm{~s}^{-1}$ and a corresponding luminosity (at the reported redshift and assuming $H_{0}=50$, $q_{0}=0$ ) of $\sim 7 \times 10^{44} \mathrm{erg} \mathrm{s}^{-1}$. We then concentrated on the second observation and first checked the MECS/PDS cross calibration constant which turned out to be $1.5 \pm 1.1$, compatible within the errors with the nominal range expected. Therefore, in the following analysis we impose that this constant varies between $0.75-0.95$, i.e. within the expected range of values. The broad band $(1-100 \mathrm{keV})$ spectrum of the source provides for the second observation a hard power law with photon index of $\Gamma=0.63_{-0.25}^{+0.21}$; furthermore marginal evidence for a line feature at around $5 \mathrm{keV}$ is also visible in the residual 


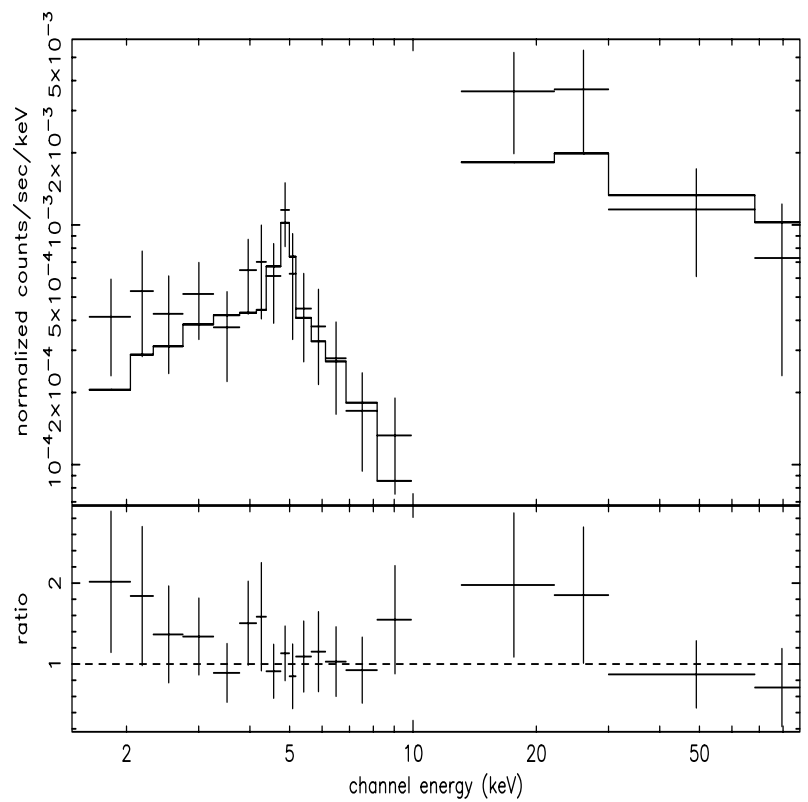

Fig. 2. BeppoSAX broad band (1-100) keV of WGA J2025.1+3342 during the second observation.

of the data to model ratio. When a Gaussian line in the rest frame of the source $(z=0.22)$ is added to the power law model, the fit slightly improves. The line is required by the data only at $72 \%$ confidence level using the F-test, is centered at $E_{\text {line }} \sim 6 \mathrm{keV}$ and has an equivalent width $E W \sim 780_{-580}^{+621} \mathrm{eV}$. The best fit of the broad band spectrum during this second observation is shown in Fig. 2. The fluxes corrected for galactic absorption are $7.4 \times 10^{-13}$ and $1.6 \times 10^{-11} \mathrm{erg} \mathrm{cm}^{-2} \mathrm{~s}^{-1}$ for the $2-10$ and $20-100 \mathrm{keV}$ band respectively; the corresponding luminosities at the reported redshift are $1.4 \times 10^{44}$ and $3.1 \times 10^{45} \mathrm{erg} \mathrm{s}^{-1}$. We therefore conclude that the source is highly luminous in X-rays and variable both in shape and intensity at these energies.

\section{Source characteristics}

In this section we analyze the broad band spectral characteristics of the source WGA J2025.1+3342 which we propose as the X-ray counterpart of 3EG J2027+3429 and show that it is indeed a new blazar gamma-ray source behind the Galactic Plane. The EGRET gamma-ray spectrum is characterized by a power law photon index $\Gamma=2.28_{-0.15}^{+0.15}$ close to the average photon index of 2.2 found in gamma-ray blazars (Mukherjee 2001). Furthermore, the EGRET light curve from the beginning to the end of the mission, obtained from the 3EG catologue (Hartman et al. 1999), clearly indicates variability of the gamma-ray flux (see Fig. 3). This was also noticed by McLaughin et al. (1996) who listed 3EG J2027+3429 as one of the galactic plane variable sources. Applying a $\chi^{2}$ test to the light curve yields a variability index of 1.19 which corresponds to a probability of $\sim 0.065$ that these data are produced by an intrinsically non variable source (McLaughin et al. 1996). The other possible gamma-ray candidate at these low galactic latitudes would be a pulsar, but this association is excluded by the measured variability. Our proposed counterpart is a strong radio source

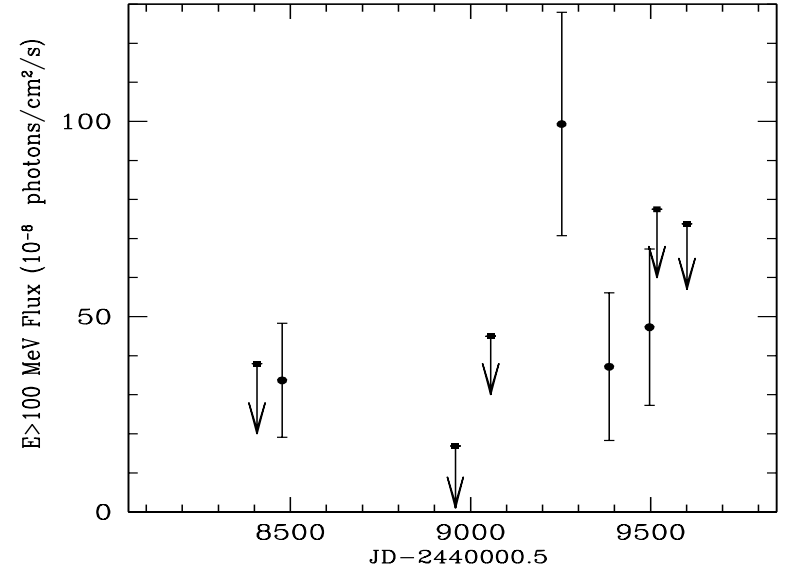

Fig. 3. Light curve of 3EG J2027+3429 from May 1991 to Jul. 1994.

detected from 0.33 to $10 \mathrm{GHz}$ (see Table 2 for details). From these broad band radio data we can estimate a power law energy index $\alpha=-0.3$ which confirms the flat spectral nature of the source (see also NED where the radio index is reported to be -0.2$)$; since FSRQ must have $\alpha \leq 0.5\left(F(v) \propto v^{-\alpha}\right)$ our source is obviously of this type.

Following Mattox et al. (1997) and Mattox et al. (2001), we find that the a-posteriori probability that the blazar we see in the EGRET error box is the correct identification is about $55 \%$. The factors that we used for this calculation are the radio flux $(2.1 \mathrm{Jy})$ and the spectral index $(+0.3)$, the $95 \%$ error radius of the EGRET source $(0.77)$, the distance of the radio source from the center of the EGRET circle $(0.91)$, and the mean distance between radio sources that are at least as strong and at least as flat as this one $\left(\sim 15^{\circ} .4\right)$. Although this value is not high enough to consider the association highly probable (Mattox et al. 1997 require at least $70 \%$ ), it is nevertheless sufficiently secure in the light of all the other observational evidence.

At optical frequencies the source is very faint and it is not reported in the USNO A2.0 catalogue (Urban et al. 1997). However, spectral data over the range 5000-10000 ̊̊ are available from the work of Sowards-Emmerd et al. (2002) and can be used to constrain the source properties.

We have also searched infrared catalogues for a counterpart at these frequencies. There are several 2 mass objects within the ROSAT error box, none of these however is related to the radio object (see Fig. 4). Taking the lowest fluxes detected in the region as our sensitivity threshold, we are able to infer an upper limit to the $J, H, K$ bands respectively. There is also an IRAS as well as an MSX (Midcourse Space Experiment) source within the X-ray error box but its positional uncertainty excludes coincidence with the radio object (see HEASARC Database). Again we can use the lowest far-infrared fluxes detected within 1 degree of our source as our sensitivity limits to infer upper limits to the emission in the $12-100 \mu \mathrm{m}$ region. All these infrared upper limits are reported in Table 3. Although not simultaneously taken, all available data are plotted in Fig. 5 in order to produce the Spectral Energy Distribution (or SED) of 3EG J2027+3429; only the 2mass and MSX upper limits are excluded since they are too close to the optical data. 
Table 2. Radio sources associated with 1WGA J2025.1+3342.

\begin{tabular}{llcclcc}
\hline \hline & Source & $\begin{array}{c}\text { RA } \\
(\mathrm{J} 2000)\end{array}$ & $\begin{array}{c}\text { Dec } \\
\mathrm{J}(2000)\end{array}$ & $\begin{array}{l}\text { Flux } \\
\mathrm{mJy}\end{array}$ & $\begin{array}{c}\text { Search offset } \\
\text { arcmin }\end{array}$ & Radio catalog \\
\hline 1 & NVSS J202510+334300 & 202510.80 & +334300.00 & $1268(20 \mathrm{~cm})$ & 0.07 & NRAO VLA sky survey catalog \\
2 & 2023+3333 & 202509.85 & +334322.60 & $2104(6 \mathrm{~cm})$ & 0.40 & 6cm Radio Catalog \\
3 & WN 2023.2+3333 & 202510.85 & +334301.40 & $982(92 \mathrm{~cm})$ & 0.07 & Westerbork northern sky survey \\
4 & TXS 2023+335 & 202510.84 & +334300.93 & $1121(82 \mathrm{~cm})$ & 0.07 & Texas Survey at $365 \mathrm{MHz}$ \\
5 & J2025+3343 & 202510.94 & +334300.21 & $2728(3.5 \mathrm{~cm})$ & 0.09 & CLASS Survey at $8.4 \mathrm{GHz}$ \\
\hline
\end{tabular}

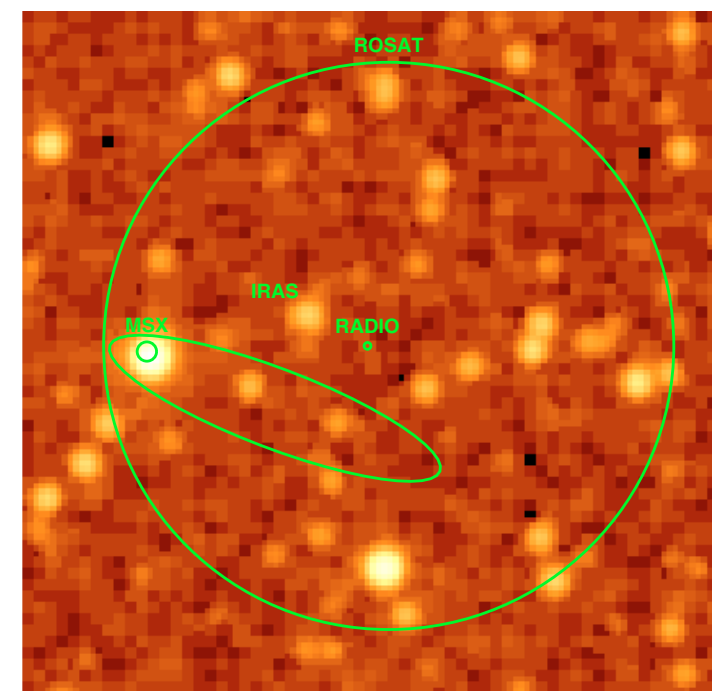

Fig. 4. 2MASS ( $J$ band) image.

In the widely adopted scenario of blazars, a single population of high-energy electrons in a relativistic jet radiate from the radio/FIR to the UV- soft X-ray by the synchrotron process and at higher frequencies by inverse Compton scattering of soft-target photons present either in the jet (synchrotron self-Compton model), in the surrounding medium (external Compton model), or in both (Ghisellini et al. 1998 and references therein). Therefore in the blazar SED, two peaks corresponding to the synchrotron and inverse Compton components should be evident. This is clearly compatible with the SED displayed in Fig. 5, where the first peak is likely located in the millimeter far-infrared region while the second most likely occurs in the soft gamma-ray range; furthermore the $\mathrm{X} /$ gamma radiation completely dominates the radiative output. Therefore, our object is consistent with being a low-frequency peaked or red blazar. These spectral characteristics (synchrotron and Inverse Compton peak at lower energies and dominance of the gammaray output) are typical of flat spectrum radio quasars and so are fully compatible with the classification of our proposed candidate.

\section{Conclusions}

From analysis of archived radio, infrared, optical and gammaray data and from our own X-ray spectroscopy, we conclude that 3EG J2027+3429 is a member of the blazar class of AGN. The X-ray source spectrum from a few $\mathrm{keV}$ up to $100 \mathrm{keV}$ is flat $(\Gamma=0.6-1.5)$ and highly variable both in intensity

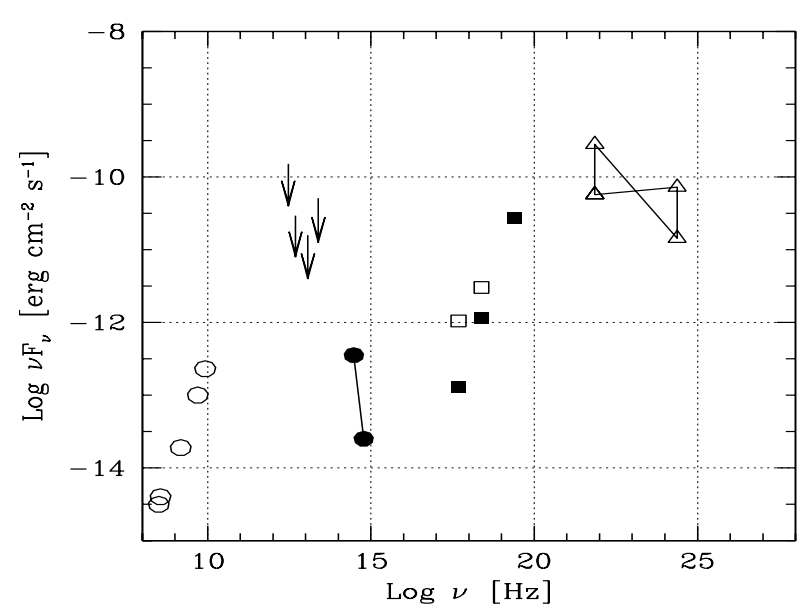

Fig. 5. Spectral Energy Distribution (SED) of 3EG J2027+3429. Open circles are radio measurements, filled circles are optical measurements, squares are our BeppoSAX observation (open squared OBS1 and filled square OBS2) and triangles are Egret measurements. The arrows indicate IRAS upper limits at $12 \mu \mathrm{m}, 25 \mu \mathrm{m}, 60 \mu \mathrm{m}$ and $100 \mu \mathrm{m}$.

Table 3. Infrared upper limit measurements.

\begin{tabular}{lll}
\hline \hline & $\begin{array}{l}\text { Wavelenght } \\
(\mu \mathrm{m})\end{array}$ & $\begin{array}{l}\text { Upper limits } \\
\left(\mathrm{erg} \mathrm{cm}^{-2} \mathrm{~s}^{-1}\right)\end{array}$ \\
\hline 1 & IRAS 12 & $5.06 \times 10^{-11}$ \\
2 & IRAS 25 & $1.57 \times 10^{-11}$ \\
3 & IRAS 60 & $2.92 \times 10^{-11}$ \\
4 & IRAS 100 & $1.50 \times 10^{-10}$ \\
5 & MSX 8 & $2.99 \times 10^{-11}$ \\
6 & 2MASS (J) 1.25 & $7.19 \times 10^{-13}$ \\
7 & 2MASS (K) 2.2 & $5.11 \times 10^{-13}$ \\
8 & 2MASS (H) 1.65 & $9.46 \times 10^{-13}$ \\
\hline
\end{tabular}

and shape. The source SED confirms the blazar nature of the source and furthermore it is compatible with its classification as a flat spectrum radio quasar. This identification is interesting because the source is in the Galactic Plane and it is the second one found in the Cygnus region. Recently, Halpern et al. (2001) identified a blazar-like radio source $\mathrm{G} 74.87+1.22$ as the counterpart of the EGRET source 3EG J2016+3657 which is also on the Galactic Plane. The probability of finding 2-3 EGRET blazars only 3 degrees from the Galactic Equator can be estimated from the total number of relatively well-identified blazars, 66, in the Third EGRET Catalog (Hartman et al. 1999). 
This implies an expectation of around two blazars within the zone $-2.4^{\circ}<b<+2.4^{\circ}$. Thus, we should not be surprised to have found another one.

Note added in proof: After acceptance of the paper for publication, we have been informed by Dr. Harvey Liszt (NRAO) that our proposed counterpart is also a strong mmwave source (B2023+336) with fluxes of 1.44, 2.8, 3.8, 2.5, 2.3 and 1.5 Jy at $1220,4590,8340,144000,22000$ and $86000 \mathrm{MHz}$ respectively; these values indicate that the source is one of the 100 or so brightest object in the sky at these wavelengths and further confirm its blazar nature.

Acknowledgements. We are grateful to Professor G.G.C. Palumbo for useful discussions and suggestions. This research has made use of SAXDAS linearized and cleaned event files produced at the BeppoSAX Science Data Center. It has also made use of data obtained from the High Energy Astrophysics Science Archive Research Center (HEASARC), provided by NASA's Goddard Space Flight Center.

\section{References}

Campana, S., Parmar, A. N., \& Stella, L. 2001, A\&A, 372, 241

Caraveo, P. 2002, the XXII Moriond Astrophysics Meeting The Gamma-Ray Universe, ed. A. Goldwurm, D. Neumann, \& J. Tran Thanh Van (The GioiPublishers)

Fiore, F., Guainazzi, M., \& Grandi, P. 1999, Handbook for BeppoSAX NFI spectral analysis, ftp://ftp.asdc.asi.it/pub/sax/ doc/software_docs/saxabc_v1.2.ps.gz or http://heasarc.gsfc.nasa.gov/docs/sax/abc/saxabc/
Fossati, G., Maraschi, L., Celotti, A., Comastri, A., \& Ghisellini, G. 1998, MNRAS, 301, 451

Frontera, F., Costa, E., Dal Fiume, D., et al. 1997, A\&AS, 122, 357

Ghisellini, G., Celotti, A., Fossati, G., Maraschi, L., \& Comastri, A. 1998, MNRAS, 301, 451

Halpern, J. P., Eracleous, M., Mukherjee, R., \& Gotthelf, E. V. 2001, ApJ, 551, 101

Hartman, R. C., Bertsch, D. L., Bloom, S. D., et al. 1999, ApJS, 123, 79

Mattox, J. R., Schachter, J., Molnar, L., Hartman, R. C., \& Patnaik, A. R. 1997, ApJ, 481, 95

Mattox, J. R., Hartman, R. C., \& Reimer, O. 2001, ApJS, 135, 155

McLaughlin, M. A., Mattox, J. R., Cordes, J. M., \& Thompson, D. J., APJ, 473, 763

Merck, M., Bertsch, D. L., Dingus, B. L., et al. 1996, A\&A, 120, 465

Mukherjee, R., Gotthelf, E. V., Halpern, J., \& Tavani, M. 2000, ApJ, 542,740

Mukherjee, R. 2001, Proceedings, vol. 558, ed. F. A. Aharonian, \& H. J. Völk (Melville, New York: American Institute of Physics), ISBN 1-56396-990-4, 324

Romero, G. E., Benaglia, P., \& Torres, D. F. 1999, A\&A, 348, 868

Sguera, V., et al. 2003, in preparation

Sowards-Emmerd, D., Romani, R. W., \& Michelson, P. F. 2002, Am. Astron. Soc. Meet., 201, 11.18

Urban, S., Corbin, T., \& Wycoff, G. 1997, A\&AS, 191, 5707

White, N. E., Giommi, P., \& Angelini, L. 1994, American Astronomical Society, 185th AAS Meeting, N.41.11; Bull. Am. Astron. Soc., 26, 1372

Zhang, L., Zhang, Y. J., \& Cheng, K. S. 2000, A\&A, 357, 957 